\title{
Time-Resolved High-Resolution Spectroscopy of roAp Stars $^{1}$
}

\section{O. Kochukhov}

Uppsala Astronomical Observatory, Sweden

T. Ryabchikova

Institute of Astronomy, Russian Academy of Sciences, Moscow, Russia Institute for Astronomy, University of Vienna, Austria

\begin{abstract}
We report results of spectroscopic monitoring of the roAp stars $\gamma$ Equ, $\alpha$ Cir and HR 3831 with the ESO 3.6-meter telescope. Series of very high-resolution and high $S / N$ spectra allowed to resolve changes of line profiles due to the pulsations. We found that pulsational behaviour of all three roAp stars is dominated by the variations of the doubly ionized rare-earth lines. Detailed analysis of the pulsational changes of Nd III and Pr III spectral features allowed us to identify the pulsational mode of $\gamma$ Equ and to study rotational modulation of the pulsational pattern in the spectra of $\alpha$ Cir and HR 3831 .
\end{abstract}

\section{Introduction}

Rapidly oscillating Ap stars belong to the group of cool magnetic chemically peculiar stars and pulsate in high-overtone $p$-modes with periods in the range of 4-16 min. Traditionally roAp stars are discovered and studied using high-speed photometric techniques. There are only a few roAp stars for which line-by-line analysis of radial velocity variations recently became available. Savanov et al. (1999) found that in $\gamma$ Equ spectral lines of $\mathrm{Pr}$ III and Nd III show the highest radial velocity (RV) amplitudes, reaching up to $1 \mathrm{~km} \mathrm{~s}^{-1}$. Thus, detailed analysis of the rapid variations of the lines of rare-earth elements (REE) can potentially provide us with crucial information about excitation of $p$-mode pulsations in roAp stars and allows spectroscopic mode identification. In order to utilize information contained in the variations of REE lines we performed very highresolution high $S / N$ time-series observations of $\gamma$ Equ, $\alpha$ Cir and HR 3831 in the 6116-6166 $\AA$ spectral region, which includes the Nd III $\lambda 6145.07 \AA$ and Pr III $\lambda 6160.24 \AA$ spectral lines. Here we provide a brief summary of the first results of our observing campaign. Detailed discussion of the oscillations in $\gamma$ Equ can be found in Kochukhov \& Ryabchikova (2001a), while preliminary results on $\alpha$ Cir and HR 3831 have been reported in Kochukhov \& Ryabchikova (2001b).

\footnotetext{
${ }^{1}$ Based on observations obtained at the European Southern Observatory (La Silla, Chile)
} 


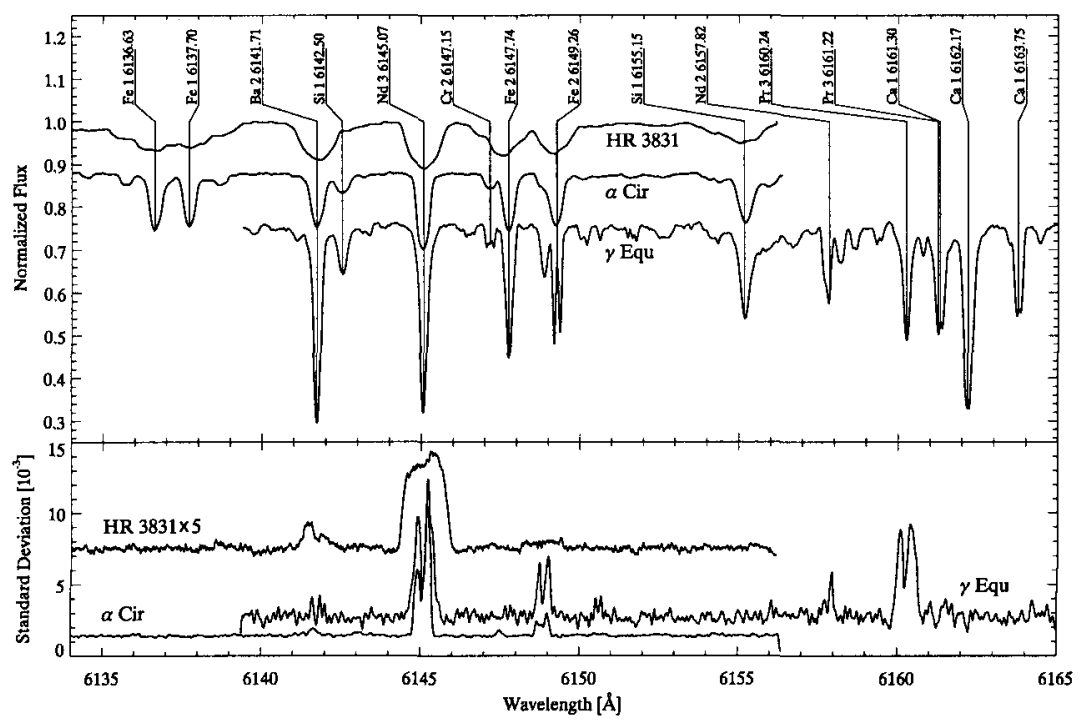

Figure 1. Comparison of the average (upper panel) and standard deviation (lower panel) spectra of roAp stars. Note that for clarity of presentation the standard deviation spectrum of HR 3831 was scaled upwards by a factor of 5 .

\section{Observations}

Observations of roAp stars were collected in July 1999 and February 2001 with the CES spectrograph at the ESO 3.6-m telescope. $\gamma$ Equ was observed during 1.5 hours using the highest resolution CES image slicer (resolution $R \simeq 170000$ ) and ESO CCD\#38. In total we obtained 31 exposures of a $26 \AA$ spectral region, centred at $\lambda 6152 \AA$. $\alpha$ Cir and HR 3831 were observed during 6 nights in a $40.4 \AA$ spectral region centred at $\lambda 6136 \AA$. The observational setup with the medium resolution CES image slicer and CCD\#61 allowed to reach resolving power $R=123000$ and record 1860 spectra of HR 3831 with a full rotational phase coverage and 290 observations of $\alpha$ Cir with incomplete coverage of the rotational cycle. Typical $S / N$ ratio of our observation lies in the range of 100 300 per pixel. The basic steps of the reduction of the spectra were performed with a set of IDL-based routines, specially adapted for the reduction of the CES spectra.

\section{Rapid spectroscopic variability of roAp stars}

We computed the standard deviation (SD) of the intensity variations for each pixel of the continuum-normalized spectra and compared it with the average observations of $\alpha$ Cir and HR 3831 in order to identify variable spectral lines. The upper panel in Fig. 1 shows average spectra of the roAp stars, while the lower panel displays SD spectra. These profiles were produced by averaging SD 

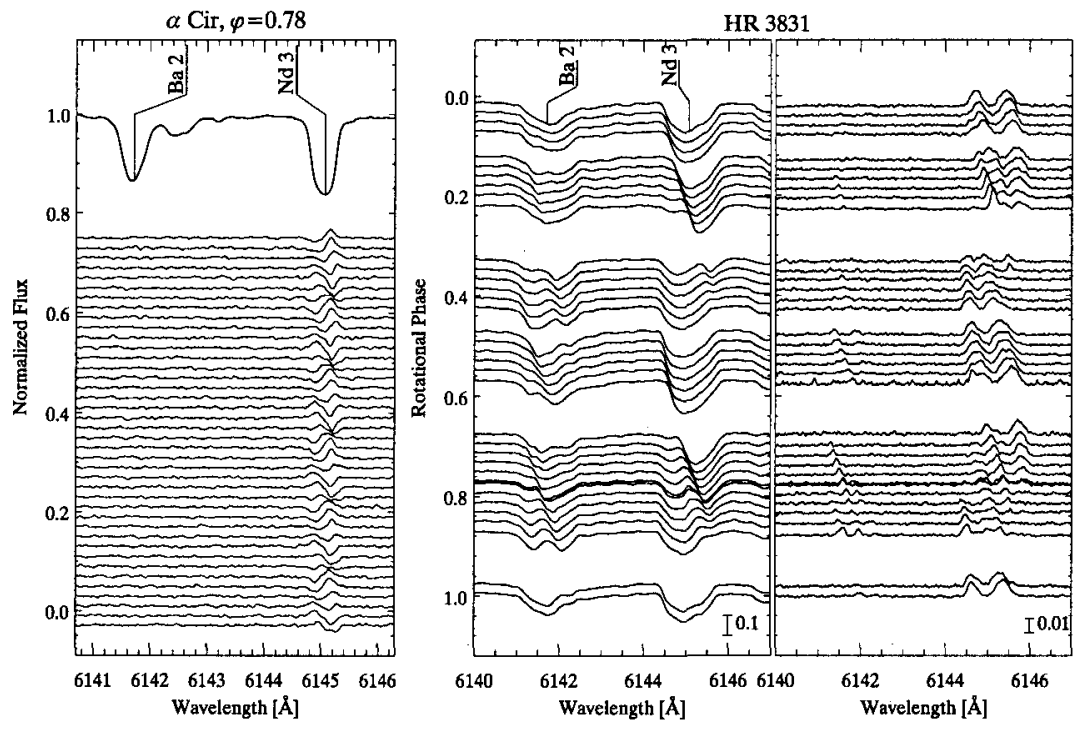

Figure 2. The left panel shows rapid changes of the Nd III $6145.07 \AA$ line profile in the spectrum of $\alpha$ Cir. The average spectrum is plotted at the top of the figure, while the difference between the average and individual time-resolved observations is plotted below. The next two panels compare rotational variability of the average $\mathrm{Nd}$ II line profile in the spectrum of HR 3831 (middle panel) with the rotational modulation of the standard deviation spectrum (right panel).

spectra computed for short sequences of time-resolved spectra and thus contain information only about spectral variability due to non-radial oscillations, but not due to rotational modulation caused by abundance spots. Analysis of the SD profiles clearly reveals outstanding variations of the Nd III $6145.07 \AA$ line in the spectra of all 3 roAp stars and Pr III $6160.24 \AA$ line in the spectrum of $\gamma$ Equ. Detailed analysis showed that the overall picture of the pulsational changes in the spectra of the 3 roAp stars is very similar: the maximal variability is observed for doubly ionized REE lines, weaker pulsations are seen for singly ionized REE, Ba II spectral features and some lines of iron-peak elements, while any variability is absent for strong lines of lighter elements (e.g. Ca I and Si I).

Ryabchikova et al. (2001) showed that abundances derived for roAp stars using Nd III and Pr III spectral lines are 1-2 dex higher than abundances obtained from lines of singly ionized $\mathrm{Nd}$ and $\mathrm{Pr}$. This spectroscopic anomaly is absent in non-pulsating Ap stars and points to an inhomogeneous vertical distribution of REE in the atmospheres of roAp stars. Combined analysis of the vertical abundance stratification and pulsational amplitudes of individual spectral features allows us to suggest that doubly ionized REE lines are formed in the atmospheric region where the pulsational amplitude reaches its maximum. The pulsational behaviour of all roAp stars analysed with high time and spectral resolution techniques is consistent with this picture and seems not to depend on 
pulsational periods, magnetic field strengths or rotation rates. This allows us to speculate that all members of the class of roAp pulsators feature similar vertical distributions of REE and pulsational amplitudes.

The discovery of large amplitude variations of doubly ionized REE lines opened a possibility to go beyond trivial time-series analysis of the RV shifts and study in detail line profile variability caused by non-radial oscillations in order to identify pulsational mode(s) and deduce other physical characteristics of the non-radial pulsations. Using a variety of mode identification techniques we estimated $\ell=2$ or $3, m=-\ell$ or $-\ell+1$ and pulsational velocity $v_{p} \approx 10 \mathrm{~km} \mathrm{~s}^{-1}$ from $\mathrm{Nd}$ III and Pr III lines in the $\gamma$ Equ spectrum.

The high quality of the observational material that we acquired for $\alpha \mathrm{Cir}$ and HR 3831 also enabled us to make a first detection of metal line profile variability induced by pulsations and to pursue detailed analysis of REE lines in the spectra of these roAp stars. In the left panel of Fig. 2 we show a one hour sequence of time-resolved $\alpha$ Cir spectra. High-amplitude rapid profile changes are evident in the difference spectra, with pulsational waves propagating from the blue to the red wing of the $\mathrm{Nd}$ III $6145.07 \AA$ line profile. (Note the absence of strong variability of the nearby Ba II $6141.71 \AA$ line.)

We observed dramatic rotational modulation of the average line profiles and pulsational pattern for HR 3831. The dense phase coverage of our observations of HR 3831 allowed us to follow in detail both modulation of rapid profile variability and changes of the average profiles caused by abundance spots. The middle and right panels in Fig. 2 illustrate how the average and the SD spectra of HR 3831 change with rotational phase. HR 3831 pulsates in the principal dipole $(\ell=1)$ non-radial mode. Its signature is clearly visible as a jump in the pattern of SD profiles of the $\mathrm{Nd}$ III line, occurring at phases 0.25 and 0.75 , as well as modulation of the RV pulsational amplitude of the same line, which changes from no detectable variability at phases 0.25 and 0.75 up to $200 \mathrm{~m} \mathrm{~s}^{-1}$ at phases 0.0 and 0.5. The observational material collected for HR 3831 gives an opportunity to reconstruct the surface distribution of chemical elements and compare it with the modulation of pulsational amplitudes. Such analysis of the relation between the inhomogeneous surface and the vertical abundance distribution, magnetic and pulsational velocity fields will be crucial for identifying the mechanism that is driving oscillations in roAp stars.

\section{References}

Kochukhov, O., Ryabchikova, T. 2001a, A\&A, 374, 615

Kochukhov, O., Ryabchikova, T. 2001b, A\&A, 377, L22

Kurtz, D.W. 1982, MNRAS, 200, 807

Savanov, I.S., Malanushenko, V.P., Ryabchikova, T.A., 1999, Astron. Letters, 25,802

Ryabchikova, T.A., Savanov, I.S., Malanushenko, V.P., Kudryavtsev, D.O. 2001, Astron. Rep., 45, 382 